\title{
Secondary motions of the shoulder during arm elevation in patients with shoulder tightness
}

\author{
Jing-Lan Yang ${ }^{\mathrm{a}}$, Tung-Wu Lu ${ }^{\mathrm{b}}$, Feng-Ching Chou ${ }^{\mathrm{b}}$, Chein-wei Chang ${ }^{\mathrm{a}}$, Jiu-jenq Lin $^{\mathrm{a}, \mathrm{c}, *}$ \\ a Department of Physical Medicine and Rehabilitation, National Taiwan University Hospital, Taipei City 100, Taiwan, ROC \\ ${ }^{\mathrm{b}}$ Institute of Biomedical Engineering, National Taiwan University, Taiwan, ROC

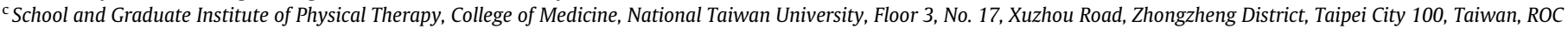

\section{A R T I C L E I N F O}

\section{Article history:}

Received 25 April 2008

Received in revised form 25 August 2008

Accepted 7 October 2008

Available online xxxx

\section{Keywords:}

Shoulder tightness

Scapula tipping

Scapular upward rotation

3D electromagnetic system

\begin{abstract}
A B S T R A C T
An analysis of secondary shoulder motions (humeral rotation, humeral head anterior/posterior translation, scapular tipping, and scapular upward/downward rotation) in subjects with anterior/posterior shoulder tightness provides the opportunity to examine the role of tightness as a means of affecting shoulder motions. Subjects with shoulder tightness (anterior, $n=12$; posterior, $n=12$ ) elevated their arms in the scapular plane. Three replicated movements were performed to the maximum motions. Kinematics data were collected by FASTRAK 3D electromagnetic system. To determine if a significant difference of the secondary motions existed between anterior/posterior shoulder tightness, two-factor mixed ANOVA models with the repeated factor of elevation angle (five elevation angles) and the independent factor of group were calculated. The relationships between the self-reported functional scores (Flexilevel Scale of Shoulder Function, FLEX-SF) and abnormal shoulder kinematics were assessed. For humeral head anterior/posterior translation, the subjects with posterior tightness demonstrated anterior humeral head translation ( $10 \mathrm{~mm}, p=0.019)$ compared to subjects with anterior tightness. The subjects with anterior tightness demonstrated less posterior tipping $\left(2.2^{\circ}, p=0.045\right)$ compared to subjects with posterior tightness. The humeral anterior translation had moderate relationships with FLEX-SF scores $(r=-0.535)$ in subjects with posterior tightness. The scapular tipping had moderate relationships with FLEX-SF scores $(r=0.432)$ in subjects with anterior tightness. In conclusion, the secondary motions were different between subjects with anterior and posterior shoulder tightness. During arm elevation, less scapular posterior tipping and less posterior humeral head translation in subjects with anterior and posterior shoulder tightness, respectively, are significantly related to self-reported functional disability in these subjects. Crown Copyright @ 2008 Published by Elsevier Ltd. All rights reserved.
\end{abstract}

\section{Introduction}

The intrinsic anatomy of the shoulder joint provides for movement with six degrees of freedom. Normal shoulder function is achieved by three-dimensional movements of the humerus and the scapula. While the humerus and scapula are moved in a defined plane or about a defined axis as the primary motion, the secondary motions, including rotations and/or translations of the humerus as well as anterior-posterior tipping and/or upward/ downward rotation of the scapula, play important roles in overall function (An et al., 1991; Harryman et al., 1992; Karduna et al., 2001; Ludewig and Cook, 2000; Rundquist and Ludewig, 2005). Coupling of external rotation of the humerus to maximal arm ele-

\footnotetext{
* Corresponding author. Address: School and Graduate Institute of Physical Therapy, College of Medicine, National Taiwan University, Floor 3, No. 17, Xuzhou Road, Zhongzheng District, Taipei 100, Taiwan, ROC. Tel.: +886 2 33228126; fax: +886233228161.

E-mail address: Jiujlin@ntu.edu.tw (J.-j. Lin).
}

vation in the scapular plane, defined as an average of $30-45^{\circ}$ anterior to the frontal plane, has been identified (An et al., 1991). Similarly, humeral head translation (a mean value of $3.79 \mathrm{~mm}$ anteriorly during flexion and $4.92 \mathrm{~mm}$ posteriorly during extension) occurs during arm elevations (Harryman et al., 1992). Additionally, coupling of posterior tipping and scapular upward rotation during arm elevations have also been described (Karduna et al., 2001; Ludewig and Cook, 2000; Rundquist and Ludewig, 2005). Shoulder motion is a result of the bony geometry, soft-tissue structures, and muscle activation.

Characteristics of coupled shoulder movements have been found to be related to the tightness of shoulder joint (Harryman et al., 1990; Ludewig and Cook, 2002; Warner et al., 1990). Increased superior translation and decreased posterior translations of the humeral head during arm elevations in subjects with posterior shoulder tightness have been related to nearer proximity between the humeral head and rotator cuff tendons, a position likely to result in impingement (Ludewig and Cook, 2002; Warner et al., 1990). Based on specimens investigation, Werner et al., spe- 
cifically, indicated that implication of the anterior part of the capsule significantly changed glenohumeral translations during arm motions (range 2 to $5.9 \mathrm{~mm}$ ) while posterior implication had little effect on translations (Werner et al., 2004). Clinically, the general aim of conservative or surgical therapy has been given to decrease shoulder tightness, which may be beneficial to decreasing clinical symptoms and/or increasing shoulder motion (Cook et al., 2003; Tyler et al., 2000). It is, however, unclear how secondary shoulder motions are affected by anterior/posterior shoulder tightness in vivo. An analysis of the patterns of secondary motions in subjects with anterior/posterior shoulder tightness provides the opportunity to examine the role of tightness as a means of affecting shoulder motions. Theoretically, clinical consequences of these altered secondary motions can include subacromial impingement, rotator cuff tendonitis, altered shoulder joint forces, and possible predisposition to degenerative changes.

Despite the impaired shoulder kinematics have been documented in patients with shoulder dysfunction, the characteristics of shoulder movement impairments in terms of shoulder tightness or the relationships between impaired shoulder movement and functional disabilities in subjects with shoulder anterior/posterior tightness have not been adequately researched. Compared to healthy subjects, patients with shoulder impingement syndrome showed decreased upward rotation $\left(4.1^{\circ}\right)$, decreased posterior tipping (average $7^{\circ}$ ), and excessive scapular elevation during simple arm elevations (Ludewig and Cook, 2000; Ludewig and Cook, 2002; Lukasiewicz et al., 1999; Madeleine et al., 1999; Szeto et al., 2005). These studies have primarily focused on the abnormal scapular kinematics to explain shoulder pathologies, such as impingement, rotator cuff injury, frozen shoulder, and work-related neck and upper limb disorders, but no studies have evaluated the shoulder movement impairments in terms of shoulder tightness or the relationships between abnormal shoulder movements and functional disabilities in patients with anterior/posterior tightness. The abnormal shoulder kinematics may not be reflected in explanations of functional disabilities in patients. For example, subjects with frozen shoulder syndrome can perform overhead activities by increasing scapular motion to compensate for limited glenohumeral joint motion (Rundquist and Ludewig, 2005). Given that altered shoulder movements may not be reflected in functional disabilities, an investigation of the relationships between impaired shoulder movements and functional disabilities helps clinicians target only those scapular movement impairments that are related to functional disabilities in patients with anterior/posterior tightness.

Shoulder tightness (ST), which is characterized by pain and functional restriction, is a common health problem (Reeves, 1975; Murnaghan, 1990; Myers et al., 2006, 2007). Reeves (1975) classified ST as two types: "idiopathic frozen shoulder" and "post-traumatic stiff shoulder". Frozen shoulder is defined as "an idiopathic contracture and loss of compliance of the glenohumeral joint capsule." Post-traumatic stiff shoulder is defined as "a limitation in humeroscapular motion associated with soft-tissue contracture after an injury." Additionally, an increased posterior shoulder tightness and glenohumeral internal rotation deficit are suggested contributors to throwing-related shoulder injuries such as pathologic internal impingement (Myers et al., 2006, 2007). ST can affect an individuals' ability to function or one's sports performance and consequently decrease quality of life.

The purpose of the study were (1) to quantitatively determine the altered secondary shoulder motions due to anterior/posterior shoulder tightness in vivo; and (2) to examine the relationships between shoulder movements and self-reported assessment scores of the affected shoulders in subjects with anterior/posterior tightness. The hypothesis was that significant secondary shoulder motion differences would exist between posterior and anterior shoulder tightness. We also hypothesized that the abnormal shoulder kinematics would reflect alterations in the patient's assessment scores of his/her ability to perform various activities of daily living.

\section{Methods}

Sample size calculations were based on detecting $5 \mathrm{~mm} / 4^{\circ}$ differences on the humeral translation/scapular tipping, which has been identified as the clinically difference between involved shoulders and control (Fayad et al., 2008; Ludewig and Cook, 2000; Ludewig and Cook, 2002). A sample size of 12 subjects per group provided $80 \%$ power to detect a clinically important difference of $5 \mathrm{~mm} / 4^{\circ}$ differences between groups, assuming a common standard deviation of $4 \mathrm{~mm} / 4^{\circ}$ and a 2 -sided hypothesis with an alpha level of .05 .

Twenty-four patients with shoulder tightness were recruited from a university hospital (Table 1 ). All subjects were at least 18 years old. The inclusion criteria for shoulder tightness were: (1) a limited ROM of a shoulder joint (ROM losses of $20 \%$ or greater compared with the noninvolved shoulder in at least two of the following shoulder motions: glenohumeral flexion, abduction, or internal/external rotation), (2) stiffness in the shoulder region for at least three months, and (3) no pain when lifting their arms. According to the measurement of shoulder tightness (Lin and Yang, 2006), twelve of the male subjects had anterior shoulder tightness (below-chest shoulder ROM $<50 \%$ of the unaffected side), while the pair-matched 12 male subjects had posterior shoulder tightness (cross-chest shoulder ROM $<50 \%$ of the unaffected side). Patients who had concomitant cervical radiculopathy, evidence of bone spurs on radiographs, or a history of traumatic injury were excluded. Each subject signed an informed consent form approved by an Institutional Review Board.

Lin and Yang have previously described the reliability (ICC $=0.82-0.91)$ and validity $\left(R^{2}=0.432\right.$, functional disabilities and tightness measurement) of the measurement of shoulder anterior/posterior tightness (Lin and Yang, 2006). In general, a fluid type inclinometer (Isomed, Portland, Oregon) was used to assess each patient's cross-chest (horizontal flexion) and below-chest (horizontal extension) shoulder ROM to represent posterior and anterior shoulder tightness, respectively, in the supine position. The humerus was passively moved into the starting position of $90^{\circ}$ of flexion and $0^{\circ}$ of adduction for assessment of posterior shoulder tightness, and the other starting position of $90^{\circ}$ of abduction for assessment of anterior shoulder tightness. While the scapula was stabilized, the humerus was then passively moved to the end of the cross-chest or below-chest ROM, as measured by the inclinometer.

Self-reported Flexilevel Scale of Shoulder Function (FLEX-SF) was used to assess the dysfunction in our patients (Cook et al., 2003). The selection of the FLEX-SF scale to assess shoulder function and disability in this study is based on its entire continuum assessment of shoulder functions and appropriate psychometric properties of reliability $($ ICC $=.90$ ) and validity (responsiveness index $=1.2$ ) (Cook et al., 2003). In this scale, respondents answer a single question that grossly classifies their level of function as low, medium, or high. They then respond to only the items that target their level of function. Scores are recorded from 1, indicating the most limited function, to 50 indicating the absence of limited function in the subject.

The sensors for the FASTRAK (Polhemus Inc., Colchester, Vermont, USA) 3D electromagnetic system were attached to the bony landmarks with adhesive tape. These surface sensor placements were the sternum, the flat superior bony surface of the scapular acromial process, and the points secured by the Velcro straps to 
Table 1

Subject Demographics.

\begin{tabular}{|c|c|c|c|c|c|c|}
\hline \multirow[t]{2}{*}{ Variable } & \multicolumn{3}{|c|}{ Subjects with anterior shoulder tightness $(n=12)$} & \multicolumn{3}{|c|}{ Subjects with posterior shoulder tightness $(n=12)$} \\
\hline & Mean & SD & Range & Mean & SD & Range \\
\hline Age (y) & 58.4 & 12.1 & $42-69$ & 53.7 & 12.6 & $34-72$ \\
\hline Weight (Kg) & 63.9 & 4.2 & $55-69$ & 61.2 & 5.2 & $52-68$ \\
\hline Height $(\mathrm{cm})$ & 163.3 & 7.3 & $161-179$ & 167.6 & 4.5 & $164-179$ \\
\hline Duration (m) & 15.3 & 6.2 & $1-24$ & 19.3 & 4.2 & $3-42$ \\
\hline Cross-chest $\left({ }^{\circ}\right)$ & $22(36)^{a}$ & 5 & $13-34$ & $10(38)^{a}$ & 7 & $5-19$ \\
\hline Below-chest $\left({ }^{\circ}\right)$ & $9(38)^{a}$ & 10 & $8-18$ & $21(42)^{a}$ & 6 & $14-30$ \\
\hline Flexion $\left({ }^{\circ}\right)$ & 137 & 18 & $90-145$ & 135 & 12 & $100-138$ \\
\hline Abduction $\left({ }^{\circ}\right)$ & 113 & 14 & $82-120$ & 109 & 10 & $82-125$ \\
\hline Int Rot $\left(^{\circ}\right)$ & 42 & 20 & $34-76$ & 16 & 8 & $10-26$ \\
\hline Ext Rot $\left(^{\circ}\right)$ & 20 & 11 & $14-26$ & 45 & 13 & $33-64$ \\
\hline FLEX-SF scores ${ }^{b}$ & 32.5 & 4.1 & $26-41$ & 34.3 & 6.1 & $23-38$ \\
\hline
\end{tabular}

a () indicates mean range of the unaffected shoulders.

b Flexilevel scale of shoulder function.

the distal humerus between lateral and medial epicondyles. A fourth sensor attached to a stylus was used to digitize palpated anatomical coordinates (bony landmarks: sternal notch, xiphoid process, seventh cervical vertebra, eighth thoracic vertebra, acromioclavicular joint, root of the spine of the scapula, inferior angle of the scapula, lateral epicondyle, and medial epicondyle. Glenohumeral joint rotation center was operationalized by anterior humeral joint and posterior humeral joint). Kinematics were collected for five seconds in the resting seated posture with arms relaxed at the sides. Subjects were then asked to perform abduction in the scapular plane oriented 40 degrees anterior to the coronal plane (Ludewig and Cook, 2000) at a self-selected comfort speed (2-3 s to complete the task). Three replicated movements were performed to the maximum motions. The high reliability (ICC $(2$, $\mathrm{k})=.91$ to .99 , similarity index $=.78$ to .97 , and less than $2^{\circ}$ standard error of measurement) of this approach has been described by Lin et al. (2005). All of the tests were performed within the recommended space after calibration (Day et al., 2000). Regarding the accuracy of in vivo measurement, Karduna et al. validated the sensor placement method with sensors fixed to pins embedded in the bone and indicated that the acromion method offered reasonably accurate representations of scapular motion (less than $3^{\circ}$ RMS error) (Karduna et al., 2001). Due to ethical issues, we did not directly measure scapular motion by fixing sensors to the scapular pins in our patients. However, potential criticisms of the surface methods were considered. The normal body-mass indices (BMI less than $23.2 \mathrm{~kg} / \mathrm{m}^{2}$ ) of our patients were intended to exclude confounding large amounts of soft-tissue. The order of tests was randomized. The dominant-right-affected shoulder was tested.

The local coordinate system developed from the digitized anatomical landmarks for the trunk and humerus was used to describe motions of the shoulder (Fig. 1) (Lin et al., 2005). Scapular orientation relative to the thorax was described using an Euler angle sequence of rotation about $Z_{s}$ (protraction/retraction), rotation about $\mathrm{Y}_{\mathrm{s}}{ }_{\mathrm{s}}$ (downward /upward rotation), and rotation about $\mathrm{X}_{\mathrm{s}}$ (posterior/anterior tipping) (Lin et al., 2005; Ludewig and Cook, 2000; Rundquist and Ludewig, 2005). Humeral orientation relative to the thorax was described using an Euler angle sequence in which the first rotation represented the plane of elevation, the second rotation defined the amount of elevation, and the third rotation described the amount of axial rotation (An et al., 1991; Lin et al., 2005; Rundquist and Ludewig, 2005). The center of the humeral head was estimated by using a helical axis method to identify the anterior-posterior translation (Ludewig and Cook, 2002; Stokdijk et al., 2000). With a humeral external fixator (bone-fixed), Ludewig and Cook assessed the accuracy of measuring humeral head translations by the surface sensor using the helical axis method as compared to bone-fixed measurement (Ludewig and Cook,
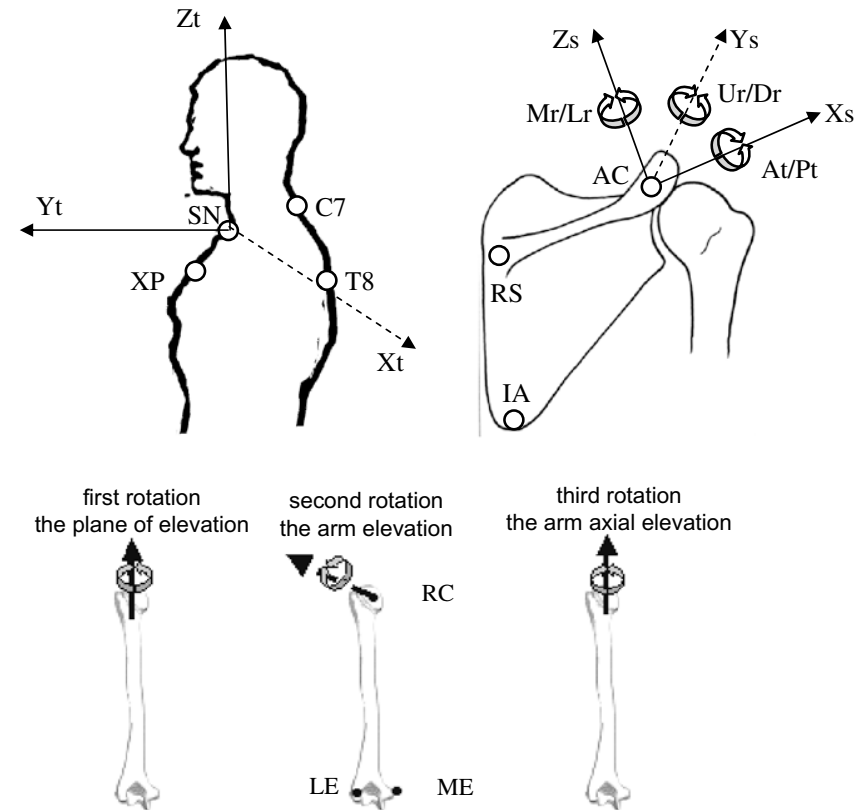
the arm elevation
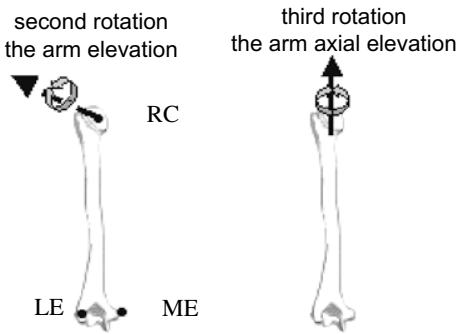

Fig. 1. Coordinate systems for the thorax, scapula, and humerus. The surface sensor placements were on the sternum inferior to the sternal notch, on the scapular acromial process, and at the point on the distal humerus between the lateral and medial epicondyles, where they were secured with Velcro straps. C7 = spinous process of the 7 th cervical vertebra, $\mathrm{T} 8=$ spinous process of the 8 th thoracic vertebra, $\mathrm{XP}=$ xiphoid process, $\mathrm{SN}=$ sternal notch, $\mathrm{RS}=$ root of the spine of the scapula, IA = inferior angle of the scapula, $\mathrm{AC}=$ acromioclavicular joint, $\mathrm{ME}=$ medial epicondyle, $\mathrm{LE}=$ lateral epicondyle, $\mathrm{RC}=$ rotation center of the glenohumeral joint, $\mathrm{At} / \mathrm{Pt}=$ anterior tipping/posterior tipping, $\mathrm{Ur} / \mathrm{Dr}=$ upward rotation/downward rotation, $\mathrm{Mr} / \mathrm{Lr}=$ medial rotation/lateral rotation. Trunk axes are aligned with cardinal planes. Xt is directed laterally, Yt is directed anteriorly, and Zt is directed superiorly. $\mathrm{Xs}$ is directed laterally from RS to AC, Ys is directed anteriorly perpendicular to the plane of the scapula, Zs is directed superiorly perpendicular to Xs and Ys.

2002). They indicated that average across the three trials, the RMS error of the surface technique was $1.5 \mathrm{~mm}$ or less.

Secondary motions were plotted versus arm elevation angle (Figs. 2 and 3). The overall characteristics of the plots were analyzed by calculating average curves based on the mean of three trials from each subject. To determine if a significant difference of the secondary motions existed between anterior/posterior shoulder tightness, two-factor mixed ANOVA models with the repeated factor of elevation angle (five elevation angles) and the independent factor of group were calculated. Bonferroni follow-up analyzes were used to adjust for multiple pair-wise comparisons. Five arm elevation angles, $30^{\circ}, 45^{\circ}, 60^{\circ}, 75^{\circ}$, and $90^{\circ}$ arm elevation, were 

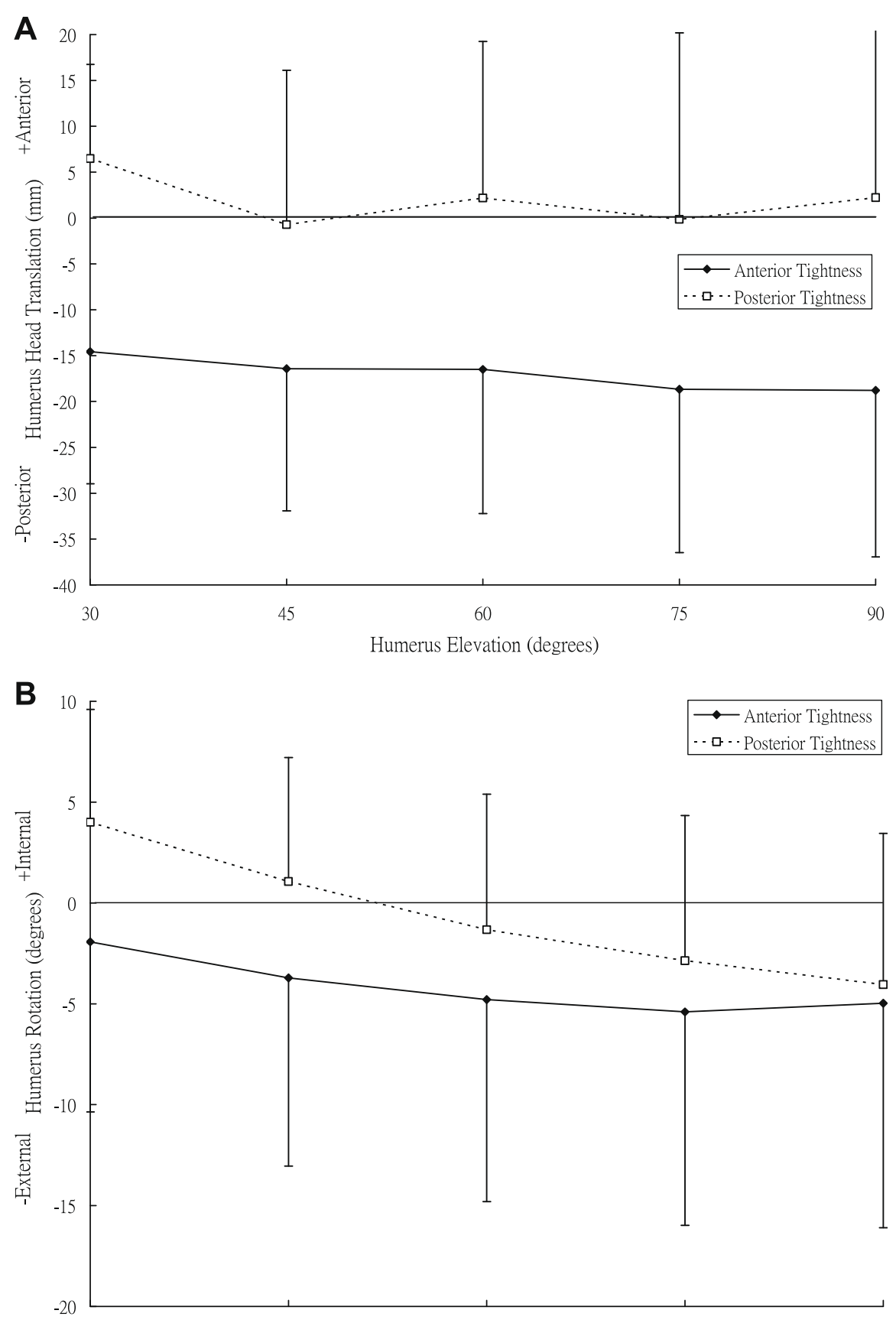

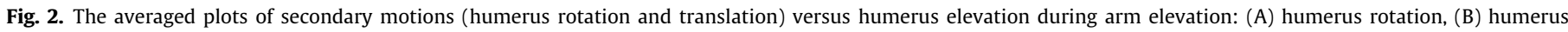
translation. Standard deviation bars represent the inter-individual variation.

selected. The corresponding secondary motions at these five arm elevation angles were tested to determine the role of tightness in affecting shoulder motions. To address potential confounding variables, we compared the planes of arm elevation between the two groups. Additionally, a covariate of humeral length was considered for translation variables using an analysis of covariance model (ANCOVA) because the size of humeral length can be the potential confounding variables.

The relationships between the scores of the FLEX-SF and abnormal shoulder kinematics (peak humeral head anterior/ posterior translation, peak humeral external rotation, peak scapular posterior tipping, and peak scapular upward rotation) were assessed by Pearson's product moment correlation coefficients in subjects with anterior/posterior shoulder tightness.

\section{Results}

The phase plots of humeral rotation, humeral head anterior/ posterior translation, scapular tipping, and scapular upward/ downward rotation angles versus arm elevation had several common features for all subjects. The common pattern was that the scapula upwardly rotated and moved toward more posteriorly tipped positions as the arm was elevated in the scapular plane. At the same time, the humerus was externally rotated throughout most of the motion, with peak external rotation occurring at $75^{\circ}$ or $90^{\circ}$ of humeral elevation. There was no difference between the two groups regarding the plane of arm elevation $\left(40.2^{\circ} \pm 0.9^{\circ}\right.$ versus $40.9^{\circ} \pm 0.5^{\circ}$.

Results from the analyzes of humeral kinematics are presented in Fig. 2. All values of shoulder kinematics passed the Shapiro-Wilk 

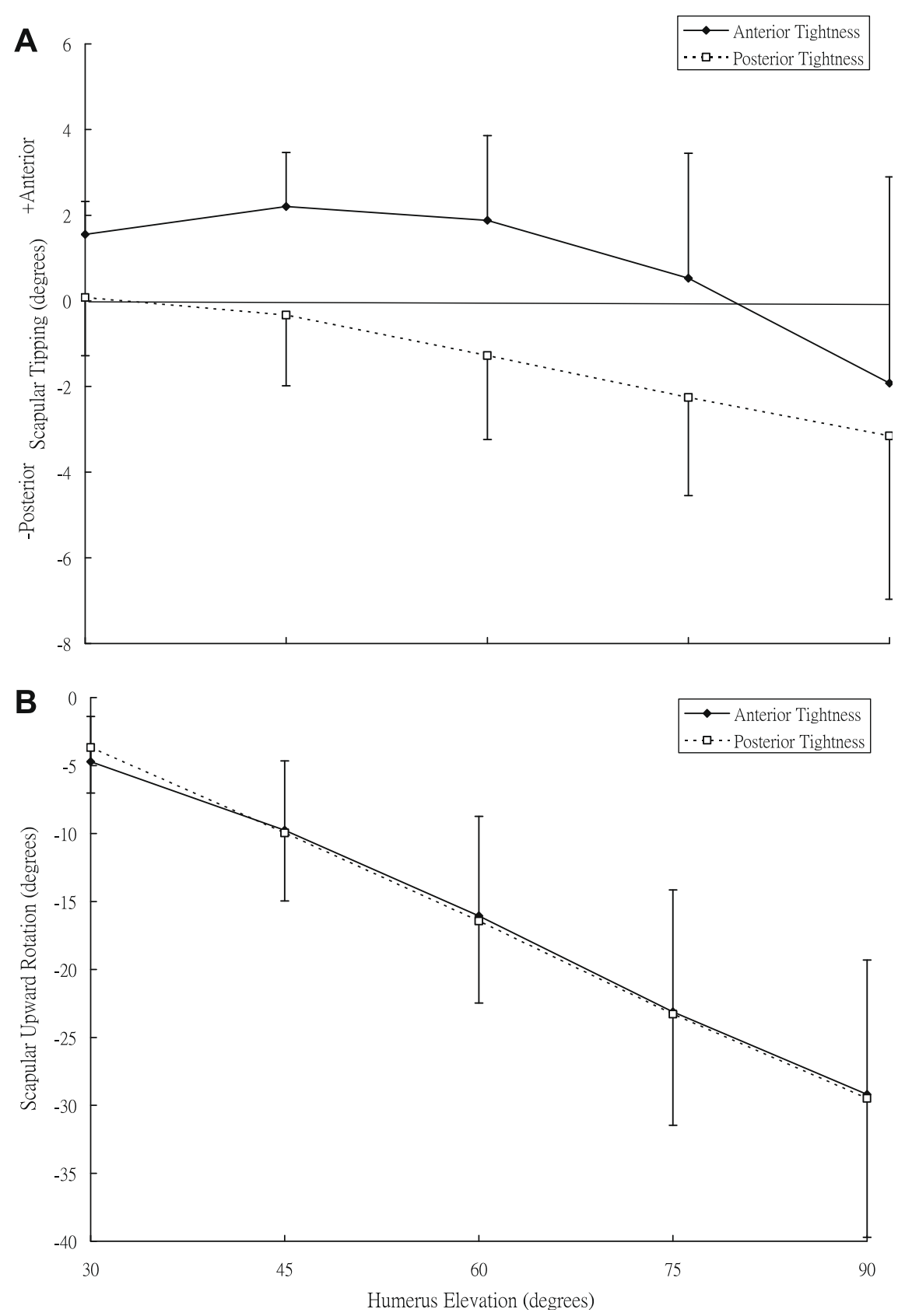

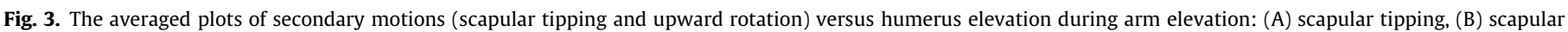
upward rotation. Standard deviation bars represent the inter-individual variation.

test $(p>0.05)$ and normality was assumed, except for scapular tipping. For humeral head anterior/posterior translation, there were significant a group main effect. The subjects with posterior tightness demonstrated anterior humeral head translation $(10 \mathrm{~mm}$, $p=0.019$ ). There was no group $\mathrm{X}$ elevation interaction effect or elevation main effect for humeral head translation. For humeral rotation, there was a significant elevation main effect. The external rotation was increased as the humerus elevated, except at $75^{\circ}$ and $90^{\circ}$ of humeral elevation. There was no group X elevation interaction or group main effect for humeral external rotation.

The analysis of scapular tipping also revealed significant group and elevation main effects (Fig. 3 ). The subjects with anterior tightness demonstrated less posterior tipping $\left(2.2^{\circ}, p=0.0045\right)$. Additionally, the scapular posterior tipping was increased as the humerus elevated, except at $30^{\circ}$ of humeral elevation. There was no group $\mathrm{X}$ elevation interaction effect for scapular posterior tipping. For the scapular upward rotation, there was a significant elevation main effect. The scapular upward rotation was increased as the humerus elevated. There was no group $\mathrm{X}$ elevation interaction or group main effect for scapular upward rotation.

Significant correlation coefficients, from -.535 to .432 , highlight the fact that shoulder kinematics (humeral translation, scapular tipping, scapular upward rotation) are related to a patient's self-reported shoulder functional activities (Table 2). Among the significant correlations, humeral anterior translation had moderate relationships with FLEX-SF scores $(r=-0.535, p=0.007)$ in subjects with posterior tightness. The scapular tipping had moderate relationships with FLEX-SF scores $(r=0.432, p=0.03)$ in subjects with anterior tightness. Scapular upward rotation correlated significantly with FLEX-SF scores in subjects with shoulder tightness 
Table 2

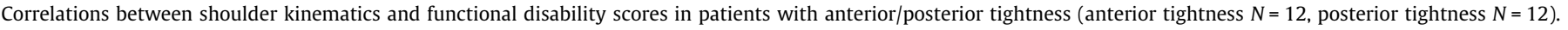

\begin{tabular}{|c|c|c|c|c|}
\hline & Humeral head anterior/posterior translation & Humeral external rotation & Scapular tipping & Scapular upward rotation \\
\hline FLEX-SF (anterior tightness) & 0.042 & 0.172 & $0.432^{*}$ & $-0.310^{*}$ \\
\hline FLEX-SF (posterior tightness) & $-0.535^{* *}$ & -0.159 & 0.222 & $-0.407^{*}$ \\
\hline
\end{tabular}

FLEX-SF: Self-reported Flexilevel Scale of Shoulder Function.

${ }^{*} p<.05$.

* $p<.01$.

(anterior tightness $r=-0.310, p=0.02$ posterior tightness $r=-0.407, p=0.03$ ).

\section{Discussion}

The results provide partial support for the hypothesis. The secondary motions were different between subjects with anterior and posterior shoulder tightness. The subjects with anterior tightness demonstrated more posterior humeral head translation $(10 \mathrm{~mm})$ and less scapular posterior tipping $\left(2.2^{\circ}\right)$ during humeral elevation as compared to the subjects with posterior tightness. These variables are also related to self-report scores. We believe the variables identified in this study, such as humeral translation, scapular tipping, and scapular upward rotation are important to consider in the rehabilitation of patients with shoulder tightness. However, the fact that the study design does not lend itself to determine whether the observed kinematic differences were a cause of pathology or simply a compensation for some other impairment should be noted.

\subsection{Effects of shoulder tightness on secondary motion}

In considering unconstrained coupled humeral head translation (average $5 \mathrm{~mm}$ ), humeral rotations (average $5^{\circ}$ ), and scapular tippings (average $3.2^{\circ}$ ) during $60-90^{\circ}$ of arm elevation in our proto$\mathrm{col}$, these movements may indicate the joint laxity that Panjabi has termed a "neutral zone". At these ranges of motion, the constraints in joint motion are tightened capsule, surface contact, and minimal muscular contraction. During the motion, a tightened posterior and anterior shoulder pushes the humeral head anteriorly and posteriorly, respectively. At the same time, a tightened anterior and posterior shoulder places the scapula in the anteriorly and posteriorly tipped positions, respectively. Our results are comparable with previous investigations (Harryman et al., 1990; Ludewig and Cook, 2002; Werner et al., 2004). Werner et al. (2004) indicated that implication of the anterior part of the capsule significantly changed glenohumeral translations during arm motions (up to $5.9 \mathrm{~mm}$ ), whereas Harryman et al. (1990) found 3-5 mm humeral head translation of cadaver specimens with motions. Ludewig and Cook (2002) found about $3^{\circ}$ of posterior tipping of the scapular during $60-120^{\circ}$ arm elevation.

\subsection{Clinical implications}

The direction of the anterior-posterior translation changes would result in the humerus being in closer proximity to the anterior undersurface of the acromion. These translation differences may result in a greater potential for impingement of the rotator cuff as the humerus is elevated. In our study, the subjects with posterior tightness had more anterior humeral head translation during humerus elevation. This finding is consistent with the posterior capsule tightness theory (Harryman et al., 1990). Harryman et al. compared humeral head translation in cadaver specimens during passive motions before and after operative tightening of the posterior capsule. After surgical tightening, the specimens showed an increase in anterior translation of 4-7 mm (Harryman et al.,
1990). Additionally, the negative correlation coefficient indicates that a subject with more severe shoulder disability (low FLEX-SF scores) had greater humeral anterior translation. It was most likely that increased posterior tightness push humeral head anteriorly that may cause impingement symptoms in the subjects with posterior tightness in our study.

The importance of scapular posterior tipping to elevate the anterior acromion during humeral elevation is provided by previous investigations (Ludewig and Cook, 2002; Lukasiewicz et al., 1999; McClure et al., 2006). These authors reported the anterior acromion and coracoacrominal ligament to be in close proximity to the rotator cuff tendon insertion in elevated positions. In our study, subjects with anterior tightness demonstrated less scapular posterior tipping during humeral elevation. Although small in magnitude $\left(2.2^{\circ}\right)$, this pattern would increase the potential for impingement. The clinical significance of these small alterations must take into consideration the limited size of the subacromial space (Warner et al., 1994). Because the data on posterior tipping did not pass the normality test, the amount of posterior tipping may be less in a specific humeral elevation range in subjects with anterior tightness (i.e., a significant interaction effect if more subjects are recruited to meet the normality test). Additionally, the positive correlation coefficient indicates that a subject with more severe shoulder disability [low FLEX-SF scores] had lower scapular posterior tipping in subjects with anterior tightness.

\subsection{Methodological considerations}

Numerous factors could introduce error in determining kinematics from an anatomical coordinate system and a skin-based marker method. The error from the palpation technique used to identify anatomical landmarks should be minimized in our study. The locations of the anatomical coordinate method were examined by a physical therapist with 15 years of experience treating musculoskeletal disorders. The coupled motions were dependent on the definition of the anatomical coordinate system. We constructed the coordinate system according to the ISB guide ( $\mathrm{Wu}$ et al., 2005). Our results are comparable with previous studies that used a similar coordinate system (Ludewig and Cook, 2002; Lukasiewicz et al., 1999; McClure et al., 2006). However, limitations in direct application of these estimates must be considered because we did not directly measure scapular motion by fixing sensors to the scapular pins in our patients. On the other hand, potential criticisms of the surface methods were considered, including BMI, our use of less than $120^{\circ}$ of elevation, and humeral length as a confounding factor. Thus, our methods should be sufficient, and our findings and interpretations are significant. Additionally, the helical axis method does not allow the tracking of the anatomical center of the humeral head during arm motion. The starting location of the humeral head is not identified with this technique. Despite its limitations, the helical method represents the minimum translation in the humeral head, which is appropriate for the instantaneous axis of the humerus during arm motion. Additionally, using the humeral length to adjust humeral head translation values, the amount of displacement (mean $=9 \mathrm{~mm}$ ) is consistent with the results from anatomical research showing that the mean ante- 
rior-posterior radius of the adult glenoid, not including the labrum, is $12 \mathrm{~mm}$ (De Wilde et al., 2004).

Interpretation of the data should be performed with caution when our sample is considered. Since the homogeneity of our sample is anterior/posterior shoulder tightness specific, the generalizability of the study results to other shoulder pathologies is uncertain. Additionally, we did not recruit normal subjects as controls. The secondary motion findings in our sample may not explain the mechanisms of other shoulder disorders.

In conclusion, description of secondary motion for the humeral head translation, humeral rotation, scapular tipping, and scapular upward/downward rotation provide a basis for the analysis of effect of shoulder tightness. Our results indicated that the secondary motions were different between subjects with anterior/posterior shoulder tightness. During arm elevation, less scapular posterior tipping and less posterior humeral head translation in subjects with anterior/posterior shoulder tightness respectively. These variables are significantly related to self-reported functional disability in these subjects.

\section{Ethical Board Review statement}

Before participating, all subjects reviewed and signed the human subject informed consent document approved by National Taiwan University Hospital.

\section{Conflict of interest statement}

None declared.

\section{References}

An KN, Browne AO, Korinek S, Tanaka S, Morrey BF. Three-dimensional kinematics of glenohumeral elevation. J Orthop Res 1991;9:143-9.

Cook KF, Roddey TS, Gartsman GM, Olson SL. Development and psychometric evaluation of the flexilevel scale of shoulder function. Med Care 2003;41:823-35.

Day JS, Murdoch DJ, Dumas GA. Calibration of position and angular data from a magnetic tracking device. J Biomech 2000;33:1039-45.

De Wilde LF, Berghs BM, Audenaert E, Sys G, Van Maele GO, Barbaix E. About the variability of the shape of the glenoid cavity. Surg Radiol Anat 2004;26:54-9.

3SPACE FASTRAK User's Manual, Revision F. Colchester, Vt: Polhemus Inc.; 1993.

Fayad F, Roby-Brami A, Yazbeck C, Hanneton S, Lefevre-Colau MM, Gautheron V, et al. Three-dimensional scapular kinematics and scapulohumeral rhythm in patients with glenohumeral osteoarthritis or frozen shoulder. J Biomech 2008;41:326-32.

Harryman II DT, Sidles JA, Clark JM. Et al: Translation of the humeral head on the glenoid with passive glenohumeral motion. J Bone Joint Surg Am 1990;72A:1334-43.

Harryman DT, Sidles JA, Harris St, Matsen FA. Laxity of the normal glenohumera jiont: a quantitative in vivo assessment. J Shoulder Elbow Surg 1992;1:66-75.

Karduna AR, McClure PW, Michener LA, Sennett B. Dynamic measurements of three-dimensional scapular kinematics: a validation study. J Biomech Eng 2001;123:184-90.

Lin JJ, Yang JL. Reliability and Validity of Shoulder Tightness Measurement in Patients with Stiff Shoulders. Man Ther 2006;11:146-52.

Lin JJ, Hanten WP, Olson SL, Roddey TS, Soto-Quijano DA, Lim HK, et al. Functional activities characteristics of shoulder complex movements: exploration with a three-dimensional electromagnetic measurement system. J Rehabil Res Dev 2005;42:199-210.

Ludewig PM, Cook TM. Alterations in shoulder kinematics and associated muscle activity in people with symptoms of shoulder impingement. Phys Ther 2000;80:276-91.

Ludewig PM, Cook TM. Translations of the humerus in persons with shoulder impingement symptoms. J Orthop Sports Phys Ther 2002;32:248-59.

Lukasiewicz AC, McClure P, Michener L, Pratt N, Sennett B. Comparison of 3dimensional scapular position and orientation between subjects with and without shoulder impingement. J Orthop Sports Phys Ther 1999;29:574-83.

Madeleine P, Lundager B, Voigt M, Arendt-Nielsen L. Shoulder muscle co-ordination during chronic and acute experimental neck-shoulder pain. An occupational pain study. Eur J Appl Physiol Occup Physiol 1999;79:127-40.

McClure PW, Michener LA, Karduna AR. Shoulder function and 3-dimensional scapular kinematics in people with and without shoulder impingement syndrome. Phys Ther 2006;86:1075-90.
Murnaghan JP. Frozen shoulder. In: Rockwood CA, Frederick A, Matsen III, editors. The shoulder. Philadelphia: WB Saunders Company; 1990. p. 837-61.

Myers JB, Laudner KG, Pasquale MR, Bradley JP, Lephart SM. Glenohumeral range of motion deficits and posterior shoulder tightness in throwers with pathologic internal impingement. Am J Sports Med 2006;34:385-91.

Myers JB, Oyama S, Wassinger CA, Ricci RD, Abt JP, Conley KM, et al. Reliability, precision, accuracy, and validity of posterior shoulder tightness assessment in overhead athletes. Am J Sports Med 2007;35:1922-30.

Reeves B. The natural history of the frozen shoulder syndrome. Scand J Rheumatol 1975;4:193-6.

Rundquist PJ, Ludewig PM. Correlation of 3-dimensional shoulder kinematics to function in subjects with idiopathic loss of shoulder range of motion. Phys Ther 2005;85:636-47.

Stokdijk M, Nagels J, Rozing PM. The glenohumeral joint rotation centre in vivo. J Biomech 2000;33:1629-36.

Szeto GP, Straker LM, O'Sullivan PB. A comparison of symptomatic and asymptomatic office workers performing monotonous keyboard work-2: neck and shoulder kinematics. Man Ther 2005;10:281-91.

Tyler TF, Nicholas SJ, Roy T, Gleim GW. Quantification of posterior capsule tightness and motion loss in patients with shoulder impingement. Am J Sports Med 2000;28:668-73.

Warner JJ, Micheli LJ, Arslanian LE, Kennedy J, Kennedy R. Patterns of flexibility, laxity, and strength in normal shoulders and shoulders with instability and impingement. Am J Sports Med 1990;18:366-75.

Warner JJ, Kann S, Maddox LM. The "arthroscopic impingement test". Arthroscopy 1994:10:224-30.

Werner CM, Nyffeler RW, Jacob HA, Gerber C. The effect of capsular tightening on humeral head translations. J Orthop Res 2004;22:194-201.

Wu G, van der Helm FC, Veeger HE, Makhsous M, Van Roy P, Anglin C, et al. International Society of Biomechanics. ISB recommendation on definitions of joint coordinate systems of various joints for the reporting of human joint motion - Part II: shoulder, elbow, wrist and hand. J Biomech 2005;38:981-92.

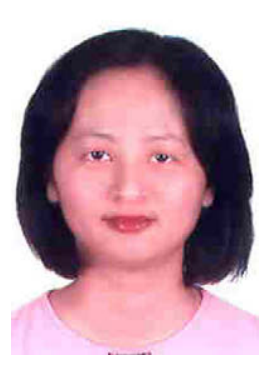

Jing-Lan Yang is a Physical Therapist in Department of Physical Medicine and Rehabilitation, National Taiwan University Hospital. She completed her Master in physical therapy in 2001, receiving it from North Carolina University in Chapel Hill, NC. Her research interests focus on physical therapy for shoulder patients.

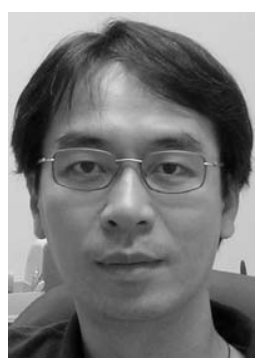

Tung-Wu Lu was born in Penghu, Taiwan in 1965. He received the $\mathrm{BE}$ degree in Civil Engineering from Tamkang University and the MS degree in Mechanical Engineering from National Chiao-Tung University, Taiwan, in 1987 and 1989, respectively. He received his D.Phil. degree in Orthopaedic Engineering from the University of Oxford, UK, in 1997. After one year working as Junior Research Fellow at St. Peter's College, Oxford University, and another year as Assistant Professor at School of Physical Therapy, China Medical University, he joined National Taiwan University where he is now Professor of Biomechanical Engineering at Institute of Biomedical Engineering and Director of NTU Rehabilitation Engineering Research Center. He is also an adjunct Professor at School of Occupational Therapy. His research interests include orthopaedic engineering, mathematical modeling of the musculoskeletal system, motion analysis, imaging biomechanics, and rehabilitation engineering.

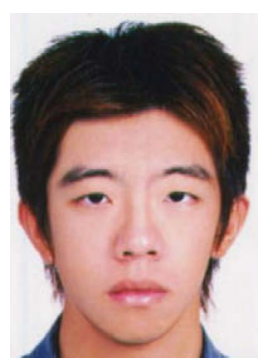

Feng-Ching Chou received his MSc degree in Biomedical Engineering in 2008 from National Taiwan University. His research interests focus on Human Motion Analysis. Currently, he is working on the application of electromyography technique to investigate mechanisms underlying work-related neuromuscular disorders. 


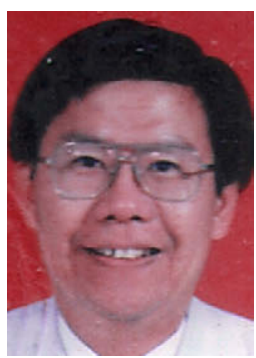

Chein-Wei Chang is a Professor in Department of Physical Medicine and Rehabilitation at National Taiwan University. He was graduated from Medical College of National Taiwan University and conferred M.D. in 1978. His research interests focus on electromyography, neurorehabilitation and clinical neurophysiology.

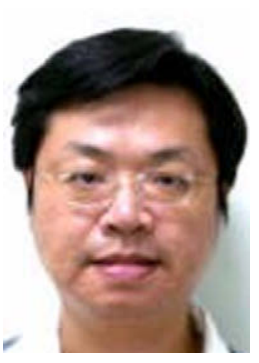

Jiu-Jenq Lin is an Associate Professor in School and Graduate Institute of Physical Therapy at National Taiwan University. He completed his Ph.D. in physical therapy in 2003, receiving it from Texas Woman's University in Houston, TX. His research interests focus on shoulder rehabilitation and characterize altered motor performance for these patients. 